\title{
Analysis of Noise Suppression Techniques using Embedded Capacitor on Split Power Bus in Multi-layer Package
}

\author{
Youchul Jeong, Joungho Kim \\ Terahertz Interconnection and Package Laboratory \\ Korea Advanced Institute of Science and Technology \\ Daejon, Korea \\ teralab@ee.kaist.ac.kr
}

\author{
Albert Chee W. Lu, Lai L. Wai, Wei Fan, \\ Boon. K. Lok, Chee K. Wong \\ Joining Technology Group \\ Singapore Institute of Manufacturing Technology \\ Singapore \\ cwlu@SIMTech.a-star.edu.sg
}

\begin{abstract}
Embedded capacitor for noise suppression on power bus is analyzed and compared with discrete capacitor by full wave simulation from $40 \mathrm{MHz}$ to $5 \mathrm{GHz}$. In this paper, the design methodology of power bus is demonstrated by analyzing self and transfer impedance characteristic of the embedded capacitor.
\end{abstract}

Keywords-component; Embedded Capacitor, Discrete Capacitor, Split Power Bus, Self-Impedance, Transfer-Impedance, Noise Coupling

\section{INTRODUCTION}

An increase in clock speed on individual chips in a system causes a large amount of current change on power bus, and this induces noise on the non-ideal power bus. This noise, known as simultaneous switching noise (SSN), is the main source of electromagnetic emissions, and nowadays the system performance is severely affected by SSN. As a result, the reduction of this noise is critical for system reliability and EMI suppression. Many useful techniques have been proposed in order to minimize the amount of SSN generated and deploying decoupling capacitor is generally one of the simplest ways to reduce the noise effectively. Basically, it reduces the loop inductances by making a short current path for the chips, and the noise, which is inversely proportional to the loop inductance, is reduced significantly. Although this decoupling capacitor scheme can be used regardless of frequency range typical discrete capacitor has parasitic and internal inductance. It exhibits inductive characteristics in the high frequency region and, therefore, it is no longer effective in suppressing SSN in the over GHz high frequency range. Although many identical capacitors can be mounted on the same board in order to reduce the effective inductance of the capacitors, a large number of capacitors reduce the reliability of the systems and occupy a large area [1]. To overcome these drawbacks of employing discrete capacitors as decoupling capacitors, an embedded capacitor using high-k dielectric thin film was proposed. The embedded capacitor uses the high-k thin dielectric film to achieve large capacitance and as it is buried inside the PCB or the package as an additional layer, it eliminates discrete capacitor pad inductance as well as the internal inductance of discrete capacitor. In addition, it also reduces the via inductance for connection to inner layers. To fully utilize the advantages presented by embedded capacitor, many different high-k dielectric materials have been developed, and many papers have been published to demonstrate their superior characteristics [2] [5]. These papers focused on the evaluation of the embedded materials with self-impedance analysis and showed the intrinsic characteristics of the embedded capacitors. In this paper, the embedded capacitor was evaluated by analyzing the self-impedance characteristic and the coupling reduction techniques between two split power planes.

\section{DESCRIPTION OF DEVICES UNDER TEST}

In this section, the layer stack-ups and layouts of the test vehicles, used for the package substrate in this paper, are described. To evaluate the decoupling performance of the embedded capacitor, the test vehicles were analyzed using both the embedded capacitor and discrete capacitor in order to compare the performance. The 3-layer test vehicle consists of signal, power, and ground layers, and the discrete capacitor is mounted on the signal layer. The discrete capacitor is connected to the power plane and the ground plane through vias. In 4-layer embedded capacitor test vehicle, additional ground plane between the signal plane and the power plane was introduced. High-k dielectric thin film material was used for the isolation between the additional ground plane and the power plane. A dielectric thickness of loum for high-k dielectric layer, which has a dielectric constant of 20 , was used. With a loum dielectric thickness, a capacitance density of $1.77 \mathrm{nF} / \mathrm{cm}^{2}$ for the embedded capacitor is achieved.

To evaluate and compare the performance of embedded capacitor, the discrete capacitors having $1 \mathrm{nF}$ capacitance and $500 \mathrm{pH}$ inductance was mounted on the 3-layer test vehicle. Reverse ratio 0612 size discrete capacitor was used due to its lower internal inductance, but it was difficult to place more than 8 capacitors due to its size and available space. Fig. 1 (a) and (b) shows the layer stack-up of 3-layer and 4-layer test vehicle with embedded capacitor respectively, and Fig. 1 (c) indicates the basic dimensions of the test vehicles. In Fig. 2, the various layouts of test vehicles according to the distribution 
and the number of vias and discrete capacitors are shown, and each DUTs is labeled with the index for easy identification.

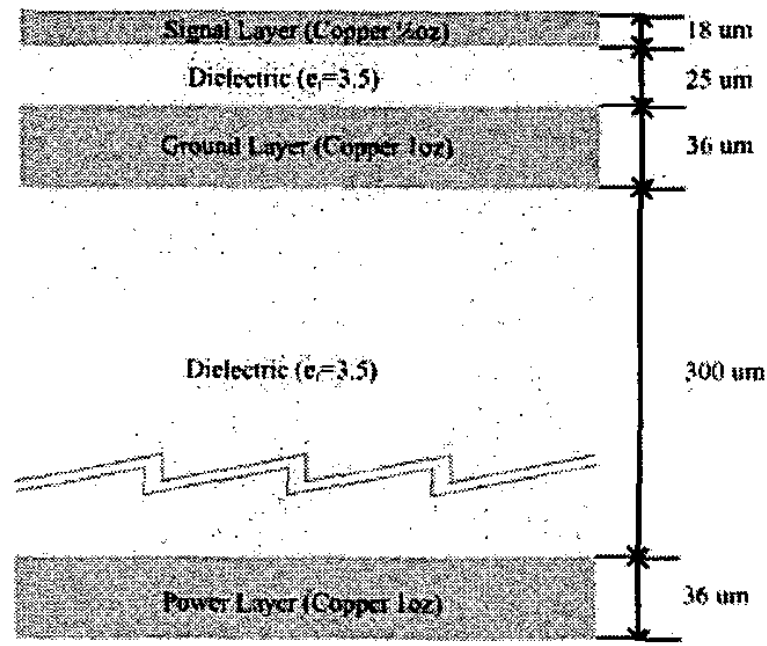

(s) Stack up low 3-layer lest vehicle

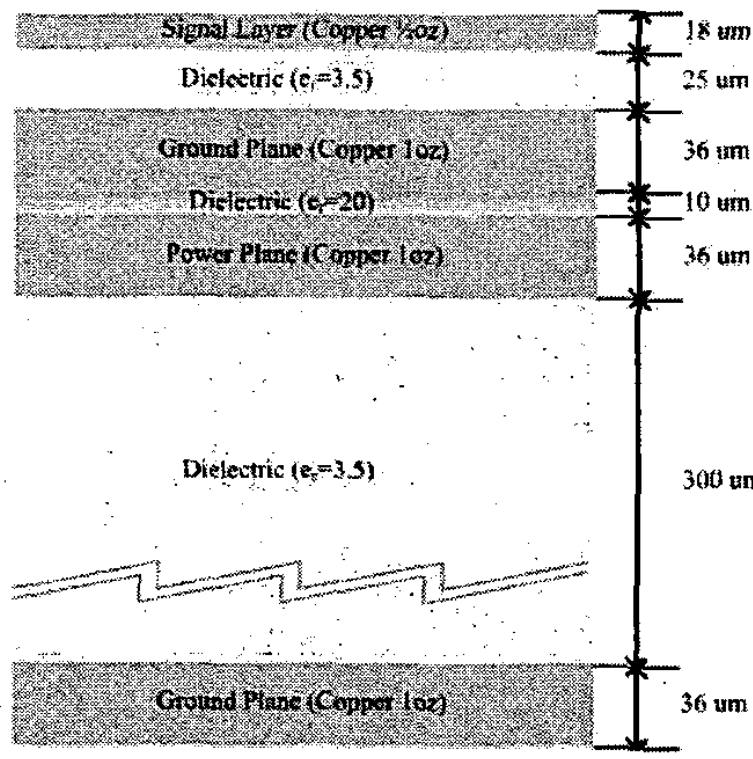

(b) Stack up for 4-byer test velicie
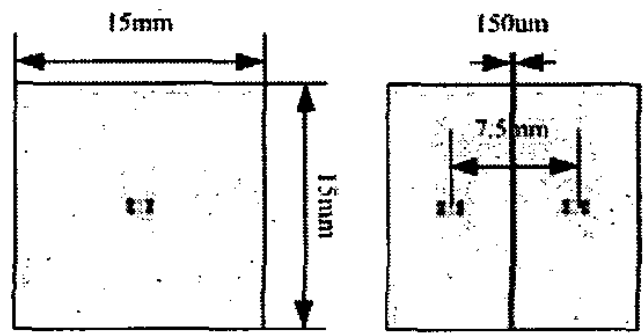

(c) Basic dimensions of tost yehicle

Fig. 1: Cross section of test vehicle
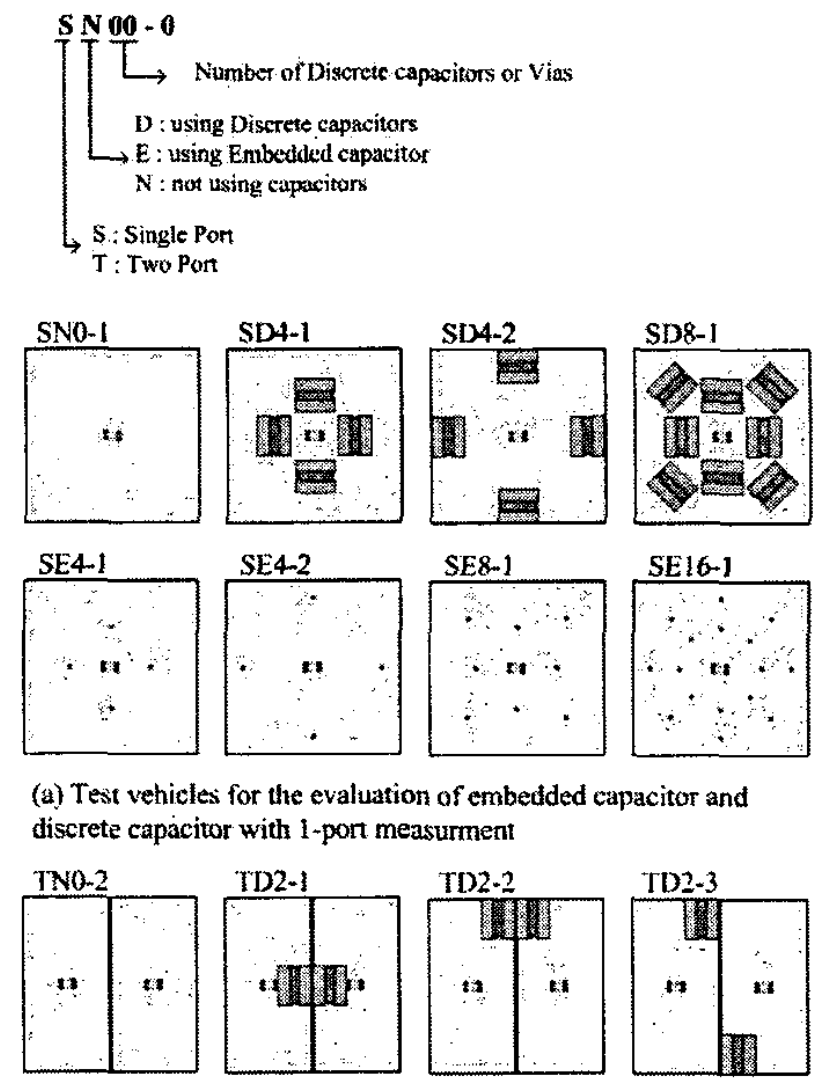

(a) Test vehicles for the evaluation of embedded capacitor and discrete capacitor with 1-port measurment
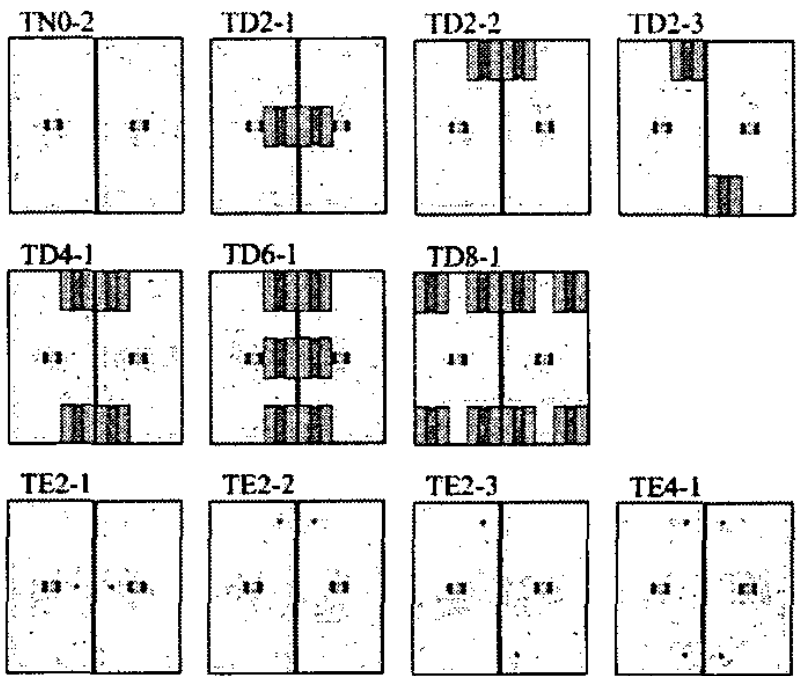

TD6-1

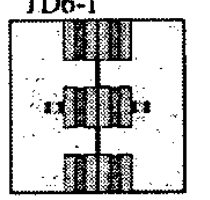

TD8-1
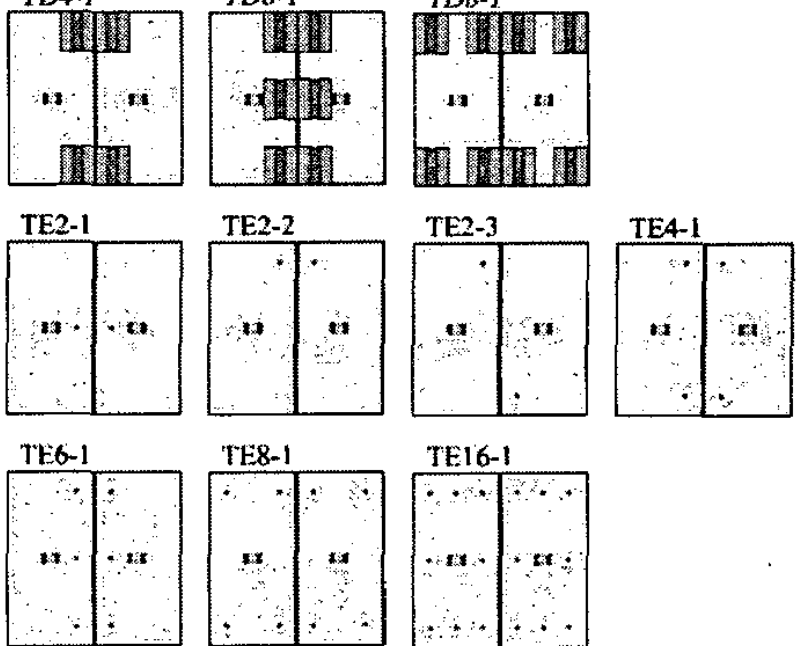

TE8-1

TE16-1
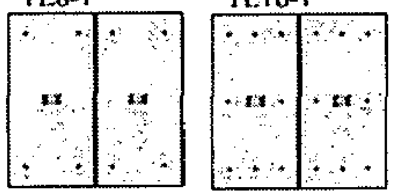

(b) Test vehicles for the analysis of coupling suppression method on split power bus using enbedded capacitor and discrete capacitor with 2-port measurement

Fig. 2: Test Vehicle Layout

The test vehicles were separated into two groups. The first group, shown in Fig. 2 (a), is used to evaluate the selfimpedance characteristics of the embedded capacitor. Power, ground and embedded layers in this group are solid planes. 
There is a single probe pad, which is used for one port analysis, in the center of package to measure the self-impedance depending on the distribution and the number of vias or capacitors. In the second group, shown in Fig. 2 (b), the power layer is split into two by a 150um gap, and there are two probe pads that are used for two port analysis in the center of each power plane. From this group, the self-impedance characteristics of the embedded capacitor with two power planes were analyzed, and the noise coupling suppression between two power planes was studied using transferimpedance behavior.

\section{SOLID POWER BUS ANAYSIS}

The self-impedance of the solid power bus was analyzed to evaluate the characteristics of the embedded capacitor using the test group designed for single port measurement, and the characteristic of the embedded capacitor is compared with that of the discrete capacitor. Fig. 3 shows the self-impedance results, and it is plotted on a linear-log scale as a function of frequency from $40 \mathrm{MHz}$ to $5 \mathrm{GHz}$.

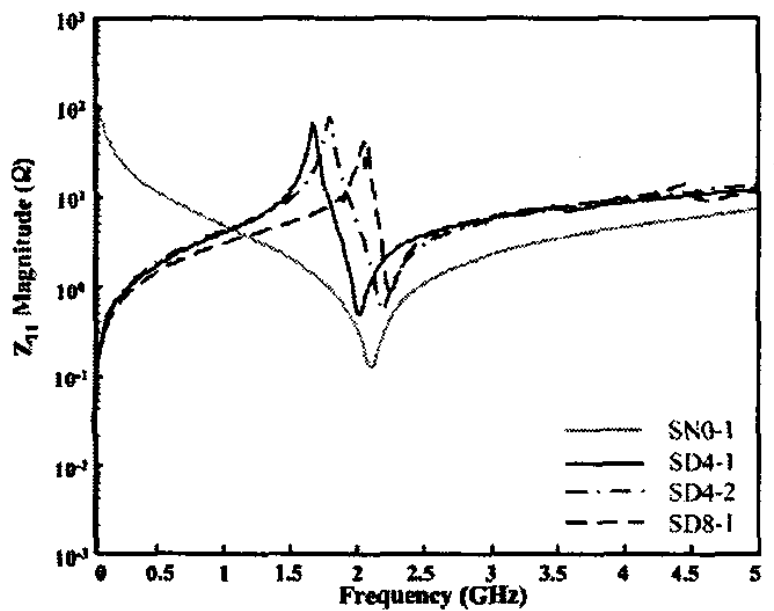

(a) Self-impedance of test vehicles using discrete capacitor

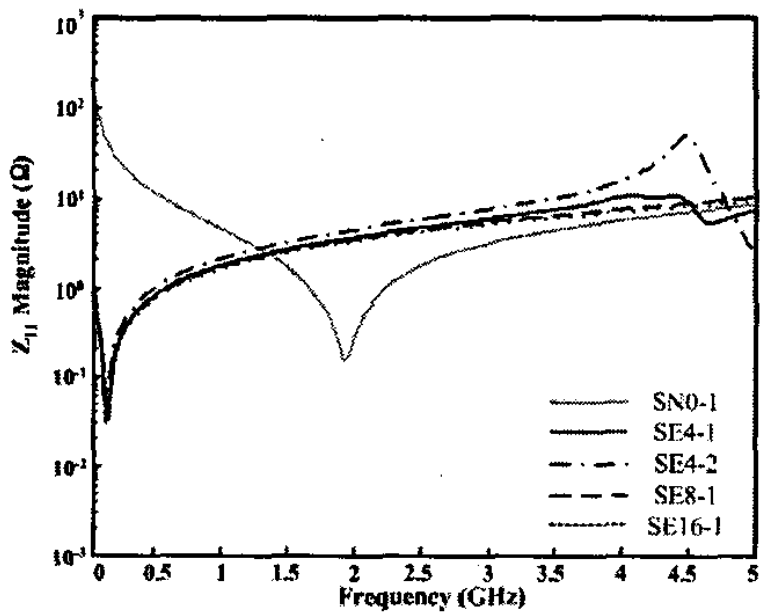

(b) Self-impedance of test yehicles using embedded capacitor

Fig. 3: Self-impedance characteristics of solid power bus

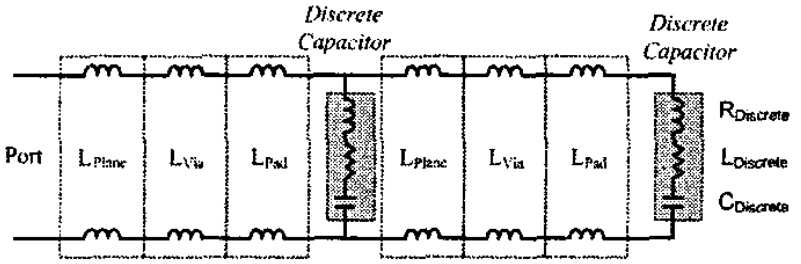

(a) Simplified power bus model with discrete capacitors

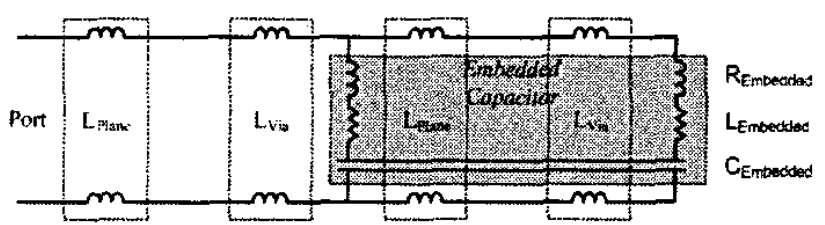

(b) Simplified power bus model with embedded capacitor

Fig. 4: Solid power bus model with each capacitor

In Fig. 3, the characteristics of the discrete capacitor and the embedded capacitor are presented by self-impedance. These self-impedance results differ depending on the capacitor type, and it is explained with the simple power bus models in Fig. 4. The following conditions are applied as the embedded capacitor is buried inside the package.

$$
\begin{aligned}
& \mathrm{L}_{\text {Embedded }} \ll \mathrm{L}_{\text {Discrete }} \\
& \mathrm{L}_{\text {via_Embedded }}<\mathrm{L}_{\text {via_Discrete }} \\
& \mathrm{L}_{\text {pad_Embedided }}=0
\end{aligned}
$$

As a result, the overall inductance of the power bus decreases with the embedded capacitor, and this not only reduce the magnitude of the self impedance in low frequency range but also shift the first parallel resonance to higher frequency. Therefore, it is able to supply low impedance to power bus over a large frequency range as shown in Fig. 3 (b). On the other hand, the effectiveness of the discrete capacitor is restricted to the low frequency region below its resonance frequency due to its large inductance.

The self-impedance is shown to be a function of the quantity and the distribution of discrete capacitors and vias, and this relationship can also be explained with the above power bus models shown in Fig. 4. The distance between discrete capacitors or vias and the probing point is proportional to the plane inductance, and the number of discrete capacitors and vias are inversely proportional to the internal inductance of each capacitor. Therefore, overall inductance of power bus is affected. The overall inductance of the power bus can be extracted from the self impedance results, and the results are summarized in Table 1.

TABLE I. EXTRACTED EFFECTIVE INDUCTANCE OF DISCRETE CAPACITORS AND EMBEDDED CAPACITORS

\begin{tabular}{|c|c|c|c|}
\hline \multicolumn{2}{|c|}{ Discrete capacitor } & \multicolumn{2}{c|}{ Embedded capacitor } \\
\hline SD4-1 & $0.53 \mathrm{nH}$ & SE4-1 & $0.28 \mathrm{nH}$ \\
\hline SD4-2 & $0.59 \mathrm{nH}$ & SE4-2 & $0.33 \mathrm{nH}$ \\
\hline SD8-1 & $0.45 \mathrm{nH}$ & SE8-1 & $0.27 \mathrm{nH}$ \\
\hline & & SE16-1 & $0.25 \mathrm{nH}$ \\
\hline
\end{tabular}


From the extracted values, it is clear that total effective inductance is strongly affected by the number of capacitors, which have larger series inductance. On the other hand, the effective inductance is kept low regardless of the number of vias in the case of the embedded capacitor due to its low selfinductance. As a result, it is possible to use the embedded capacitor in higher frequency region with a minimum number of vias. Furthermore, although it is difficult to use more than 8 discrete capacitors on the test package due to limited space constraint, it is possible to add more vias, which will further reduce the inductance, in the package for the embedded capacitor application.

\section{SPLIT POWER BUS ANALYSIS}

In this section, the inter-plane coupling between two power planes was analyzed by studying the self-impedance at each port and transfer-impedance between two ports. When a power plane is separated into two planes by a gap, the noise coupling is reduced significantly. However, it is not sufficient for the noise sensitive circuit such as PLL although the decoupling. capacitors can be used in this application. Discrete capacitor and the embedded capacitor characteristics were analyzed with respect to the distribution and the number of capacitors and vias. Fig. 5 shows the self-impedance and transfer-impedance characteristics for various configurations.

In order to analyze the relationship between the distribution patterns and the performance of the power bus, the number of discrete capacitors and vias was fixed at two. The selfimpedance and the transfer-impedance plots are then compared. Firstly the distance of discrete capacitors and vias from the probing point was studied. In TD(E)2-1, the distance is very short compare to the other test vehicles and the same distance can be observed in TD(E)2-2 and TD(E)2-3. As stated in section III earlier, when the discrete capacitors and the vias are located near the probe pad, the impedance has the lowest value because of smaller plane inductance. Therefore, TD(E)-1 exhibits the lowest inductance because the vias are placed closer to the probe pad than in the other test vehicles and the transfer impedance is also the lowest because the transfer impedance is highly correlated with the self-impedance. However, it is usually impractical to place the discrete capacitors or the vias in the center of the package as the area must be reserved for chip mounting and routing. Hence, the distribution pattern shown in TD(E)2-2 and TD(E)2-3 are analyzed. In $T D(E) 2-2$ and $T D(E) 2-3$, each via is located on different power planes at the same end of the split power planes next to the slot and diagonally at the opposite end of the split power planes respectively. Fig. 6 shows the return current loop and impedance map for both cases. Although each via is located on different power planes, both of them supply return current path for each port, and it reduces the overall inductance as shown in Fig. 6 (a). There are no appreciable differences in 1-dimensional current loop between the two test vehicles. However, it can be seen from a 2-dimensional impedance map illustrated in Fig. 6 (b) that each distribution is quite different. If it is assumed that the discrete capacitors or the vias are located at port 3 and port 4 (or port 4 ), the following formula can be derived, where $Z_{d}$ is the impedance of decoupling capacitor.

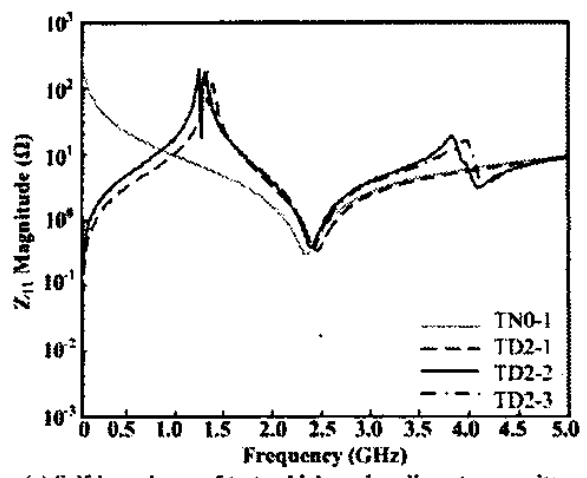

(a) Self-lmpedance of test vehicles using discrete capacitor

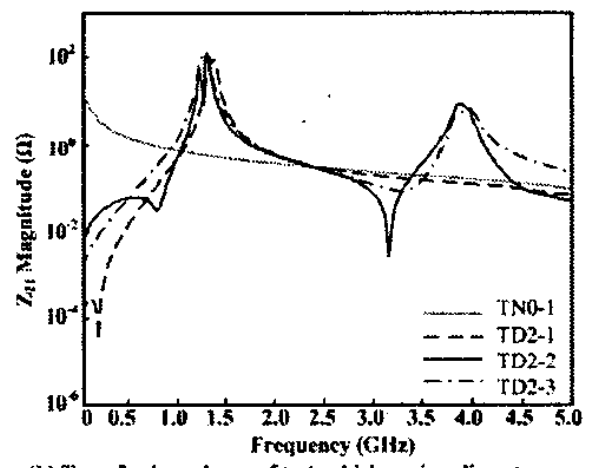

(b) Transfer-imperfance of test vehicles using discrete capacitor

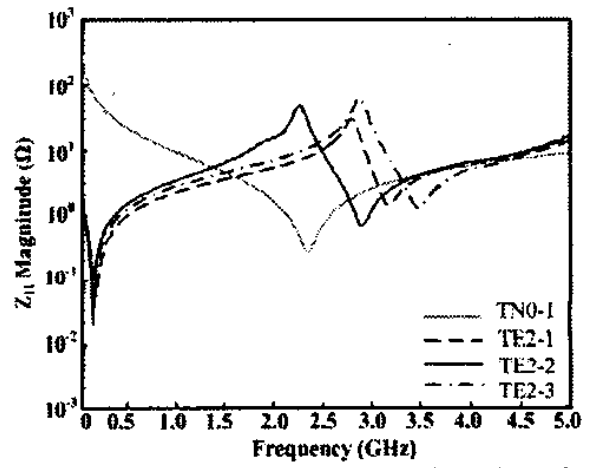

(c) Self-impedance of test vehicles using embedded capacitor

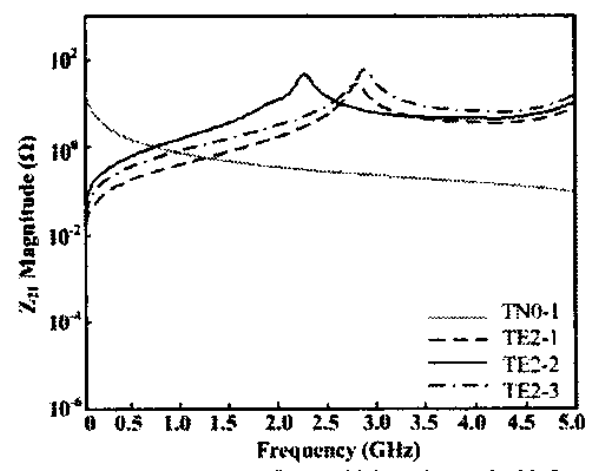

(d) Transfer-impedance of test vekicles using embedded capacitor

Fig. 5: Impedance characteristics depending on the distribution of capacitors and vias 


$$
\begin{aligned}
& Z_{11_{-} \text {with_capacitorsor vias }}=Z_{11}-\frac{2 Z_{13}^{2}}{Z_{33}+Z_{34}+Z_{d}} \\
& Z_{21_{-} \text {with_capacitorsor vias }}=Z_{21}-\frac{2 Z_{13} Z_{23}}{Z_{33}+Z_{34}+Z_{d}}
\end{aligned}
$$

It can be seen that $Z_{34}$ is the only variable and the other impedances are constants. The impedances in the right side of equations are the self-impedance and the transfer- impedance at each port. Comparing $Z_{34}$ with $Z_{34}^{\prime}$ in Fig. 6, which indicate the transfer-impedance between decoupling capacitors in $T D(E) 2-2$ and $T D(E) 2-3$ respectively, the magnitude of $Z^{\prime}{ }_{34}$ is larger than that of $Z_{34}$ because the transfer-impedance is dominated by the plane inductance. The self-impedance and the transfer-impedance in the power bus decreases as $Z_{34}$ increases. As a result, TD(E)2-3 exhibits the lower impedance characteristics than TD(E) 2-2 and it has been validated with the impedance results in Fig. 5.

Secondly the number of discrete capacitors and vias is varied, and the impedance plots are shown in Fig. 7. As expected, the increase in number of capacitor and via causes inductance to reduce, and as a result, the series and first parallel resonance frequencies shift to higher frequency. As shown in the previous section, the inductance is inversely proportional to the number of vias, and the best performance is illustrated by TD8-1 and TE16-1.

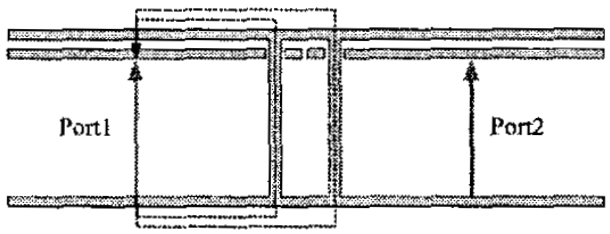

(g) 1-dimensional return current loop

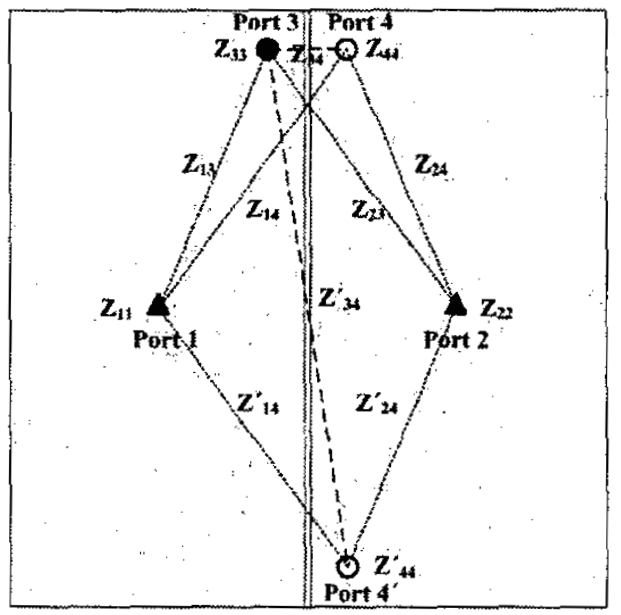

(b) 2-dinensional impedance map

Fig. 6: Return current loop in cross section and impedance map

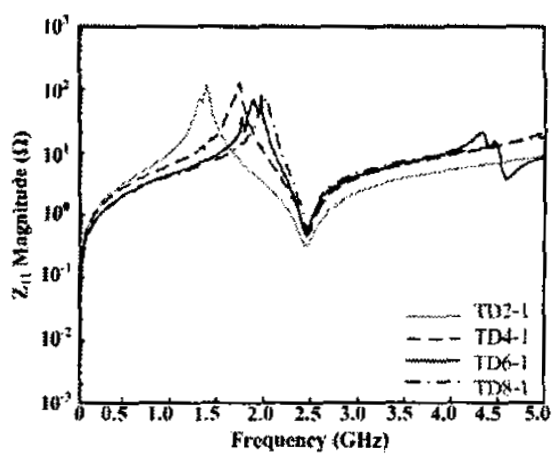

(a) Seff-inpedance of test vehicles using discrete capacitor

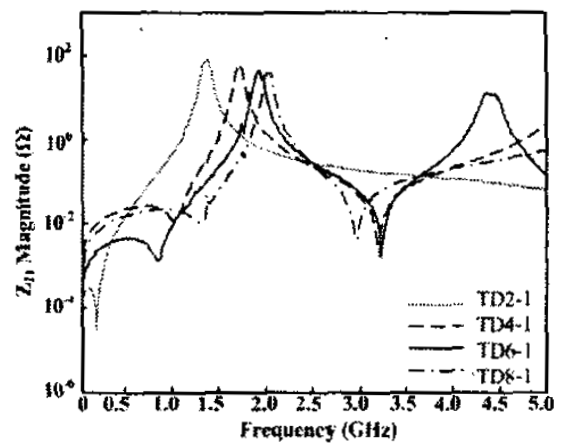

(b) Transfer-impetance of test vehlcles using discrete capacitor

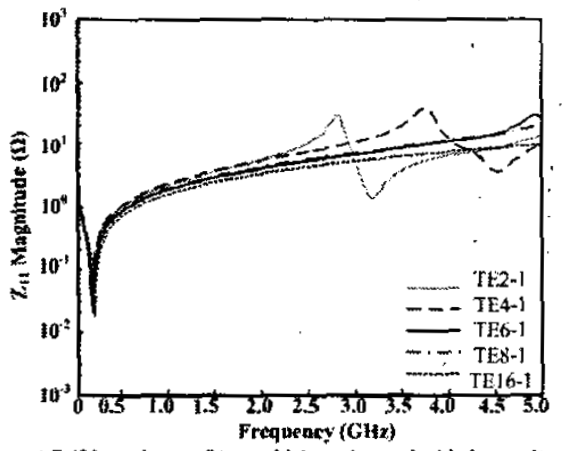

(c) Self-inpedance of test vebicles using embedded capacitor

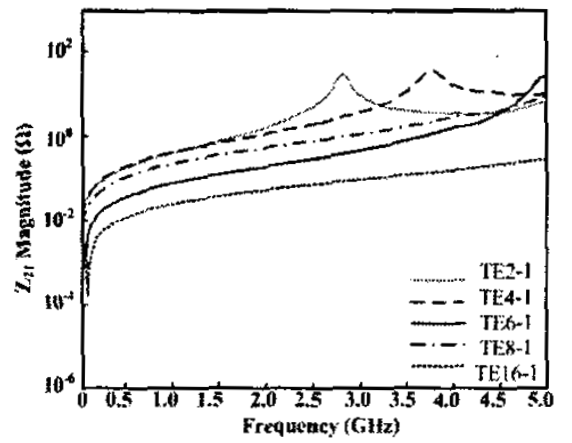

(d) Transfer-impedance of test vebicles using embedded capacitor

Fig. 7: Impedance charactersitics depending on the distribution of capacitors and vias

Effective inductance was also extracted and it is summarized in table II. It can be seen there is a significant 
reduction in inductance exhibited by embedded capacitor compared to discrete capacitor.

TABLE II. EXTRACTED EFFECTIVE CAPACITANCE AND INDUCTANCE OF THE EMBEDDED CAPACITOR

\begin{tabular}{|c|c|c|c|}
\hline \multicolumn{4}{|c|}{ Effective inductance } \\
\hline Discrete capacitor & \multicolumn{2}{|c|}{ Embedded capacitor } \\
\hline TD2-1 & $0.91 \mathrm{nH}$ & TE2-1 & $0.35 \mathrm{nH}$ \\
\hline TD2-2 & $1.37 \mathrm{nH}$ & TE2-2 & $0.48 \mathrm{nH}$ \\
\hline TD2-3 & $1.37 \mathrm{nH}$ & TE2-3 & $0.41 \mathrm{nH}$ \\
\hline TD4-1 & $0.80 \mathrm{nH}$ & TE4-1 & $0.37 \mathrm{nH}$ \\
\hline TD6-1 & $0.61 \mathrm{nH}$ & TE6-1 & $0.30 \mathrm{nH}$ \\
\hline TD8-1 & $0.47 \mathrm{nH}$ & TE8-1 & $0.26 \mathrm{nH}$ \\
\hline & & TE16-1 & $0.26 \mathrm{nH}$ \\
\hline
\end{tabular}

\section{CONCLUSION}

In this paper, it was demonstrated that the embedded capacitor can be used effectively for noise coupling suppression. The characteristic of the various embedded capacitors was analyzed based on the full wave simulation and the measurement results. From the analysis, it is very clear that the embedded capacitor has lower inductance than the discrete capacitor and it is most suitable for power/ground noise reduction.

After evaluating the characteristic of the embedded capacitor, various techniques were analyzed to suppress noise coupling using the embedded capacitor based on the selfimpedance and the transfer-impedance results. From this analysis, the relationship between the distribution of vias and the noise coupling suppression was developed.

\section{ACKNOWLEDGMENT}

Authors would like to acknowledge the collaborative research project funding and support from Singapore Institute of Manufacturing Technology, A*STAR, and Korea Advanced Institute of Science and Technology, Terahertz Media and System Laboratory (Dept of EE and CS)

\section{REFERENCE}

(1) Larry D. Smith, Raymond E. Anderson, Douglas W. Forehand, Thomas J. Pelc, and Tanmoy Roy, "Power Distribution System Design Methodology and Capcitor Selection of Modern CMOS Technology", IEEE Trans on AP, vol 22, no. 3, August, 1999, pp. 284-291.

[2] Hyungsoo Kim, Youchul Jeong, Jongbae Park, SeoKyu Lee, JongKuk Hong, Yongsoo Hong, and Joungho Kim. "Significant Reduction of Power/Ground Inductive Impedance and Simultaneous Switching Noise by Using Embedded Film Capacitor", Electrical Performance of Electronic Packagin, Oct, 2003, pp. 129-132.

[3] An Mandou and Luc Martens, "Electrical Behavior of Decoupling Capacitors Embedded in Multilayerd PCBs", IEEE Trans. on EMC, vol 43 , no. 4, November 2001, pp. 579-556.

[4] Minjia Xu, and Todd H. Hubing, "Estimating the Power Bus Impedance of Printed Circuit Boards with Embedded Capacitnace", IEEE Trans. on AP, vol. 25, issue 3, Aug, 2002, pp.424-432.

[5] Minjia Xu, Todd H. Hubing, Juan Chen, Thomas P. Van Doren, James L Drewniak, and Richard E. Dubroff, "Power-Bus Decoupling with Embedded Capacitance in Printed Circuit Board Design", IEEE Trans. n C, vol 45, issue 1, Feb, 2003, pp. 22-30. 